\title{
Delayed tumour enhancement on gadoxetate-enhanced MRI is associated with overall survival in patients with colorectal liver metastases
}

\author{
Helen M. C. Cheung ${ }^{1} \cdot$ Paul J. Karanicolas ${ }^{2} \cdot$ Natalie Coburn $^{2} \cdot$ Vikrum Seth $^{1} \cdot$ Calvin Law $^{2} \cdot$ Laurent Milot $^{1}$
}

Received: 2 May 2018 / Revised: 11 June 2018 / Accepted: 19 June 2018 / Published online: 10 July 2018

(C) The Author(s) 2018

\begin{abstract}
Objectives To determine whether tumour enhancement on preoperative delayed-phase gadoxetate-enhanced MRI can predict long-term survival in patients with colorectal liver metastases (CRCLM) post-hepatectomy.

Materials and methods Sixty-five patients who received a preoperative gadoxetate-enhanced MRI prior to liver resection for CRCLM from January 1, 2010, to December 31, 2012, were included in this retrospective study. Target tumour enhancement (TuEn) was calculated as the mean percentage increase in SNR from precontrast to 10-min or 20-min delayed phase for up to two target lesions. Per-patient TuEn was stratified into weak and strong enhancement based on the cut-off determined by the Youden Index for 3-year survival. Kaplan-Meier and Cox regression analyses were used to determine whether tumour enhancement could predict overall survival independent of potential confounders (clinical risk score).

Results The proportion surviving at 3 years was $85.1 \%$ in patients with strong TuEn at $10 \mathrm{~min}$ vs. $56.5 \%$ in those with weak TuEn at $10 \min (p=0.001)$. The proportion surviving at 3 years was $79.4 \%$ in patients with strong TuEn at $20 \mathrm{~min}$ vs. $58.7 \%$ in those with weak TuEn at $20 \mathrm{~min}(p=0.011)$. After adjusting for potential confounders, the hazard ratio of death was $0.24(p=0.009)$ in patients who had weak TuEn at $10 \mathrm{~min}$ and $0.32(p=0.018)$ in patients who had weak TuEn at $20 \mathrm{~min}$.

Conclusions Strong delayed tumour enhancement seen on gadoxetate-enhanced MRI is associated with overall survival in patients with CRCLM post-hepatectomy and may be useful for preoperative risk stratification.

Key Points

- Delayed tumour enhancement of colorectal liver metastases on gadoxetate-enhanced MRI is associated with survival post-hepatectomy

- Delayed tumour enhancement of colorectal liver metastases on gadoxetate-enhanced MRI can be measured at both 10 min and 20 min post-contrast injection.
\end{abstract}

Keywords Colorectal cancer $\cdot$ Neoplasm $\cdot$ Metastases $\cdot$ Gadolinium $\cdot$ Survival

\section{Abbreviations \\ CEA Carcinoembryonic antigen \\ CRCLM Colorectal liver metastases \\ MRI Magnetic resonance imaging}

Helen M. C. Cheung

hmc.cheung@mail.utoronto.ca

1 Department of Medical Imaging, Sunnybrook Health Sciences Centre, University of Toronto, 2075 Bayview Avenue, Rm AB 202, Toronto, ON M4N 3M5, Canada

2 Department of Surgery, Sunnybrook Health Sciences Centre, University of Toronto, 2075 Bayview Avenue, Toronto, ON M4N 3M5, Canada
PACS Picture archiving and communication system

SNR Signal-to-noise ratio

SNR0 Signal-to-noise ratio on precontrast phase

SNRX Signal-to-noise ratio at " $x$ " minutes post contrast

TuEn10 Tumour enhancement 10 min post contrast

TuEn20 Tumour enhancement 20 min post contrast

TuEnX Tumour enhancement at " $\mathrm{x}$ " minutes post contrast

\section{Introduction}

Colorectal cancer is the third leading cause of cancer deaths worldwide (after lung and liver) [1]. Most of the deaths are related to metastatic disease, with the liver being the most 
common site for metastases. With improvements in surgical techniques and systemic therapy in the last 2 decades, the survival for colorectal liver metastases (CRCLM) has significantly improved. In patients who are candidates for surgery, 5 -year survival rates are in the range of $25-40 \%$ [2, 3].

It has recently been shown that late gadolinium enhancement of CRCLM on preoperative magnetic resonance imaging (MRI) with an extracellular contrast agent, gadobutrol, is associated with tumour fibrosis and overall survival post-hepatectomy [4]. Late gadolinium enhancement of CRCLM on MRI with gadobutrol may be associated with tumour fibrosis due to leakage of extracellular contrast into the tumour via the interstitium [4]. It has previously been established in the histology literature that tumour fibrosis is associated with good outcomes post-hepatectomy [5, 6].

Although MRI with extracellular contrast agents is still commonly used in many institutions, others now use MRI with hepatobiliary-specific contrast agents, such as gadoxetate (gadoxetic acid), to stage CRCLM [7]. Gadoxetate is known to have a dual mechanism: in addition to hepatobiliary-specific uptake, gadoxetate also has an extracellular component. Therefore, we would expect that CRCLMs would also demonstrate delayed enhancement on gadoxetate-enhanced MRI via its extracellular component. In fact, several previous papers have described late gadolinium enhancement of CRCLMs on delayed phase imaging [8, 9].

Although there is currently no literature on the timing of delayed phase imaging and correlation with tumour fibrosis in CRCLM, prior studies in the cardiac literature demonstrated that late gadolinium enhancement is best correlated with tissue fibrosis between 10-30 min using extracellular contrast agents [10]. Given that delayed phase imaging on routine liver MRI with gadoxetate is typically performed at $10 \mathrm{~min}$ and $20 \mathrm{~min}$ post-contrast, measurements of late gadolinium enhancement were performed at both of these time points [7].

Therefore, the goal of this study was to determine whether tumour enhancement of CRCLM seen on preoperative gadoxetate-enhanced MRI is associated with long-term survival post-hepatectomy at $10 \mathrm{~min}$ and $20 \mathrm{~min}$ post-contrast injection.

\section{Materials and methods}

This retrospective study was approved by the institutional research ethics board, which waived the requirement for informed consent. Patients with CRCLM who received a preoperative MRI with gadoxetate (Primovist ${ }^{\mathrm{TM}}$, Eovist ${ }^{\mathrm{TM}}$ ) prior to hepatectomy from January 1, 2010, to December 31, 2012, at a single tertiary cancer centre were included in the study. Patients were excluded if they received portal vein embolisation prior to the MRI or if images were of unacceptable quality for analysis.
MRIs were obtained for clinical purposes (diagnosis or staging and/or preoperative planning). As per institutional clinical guidelines for liver MR imaging, patients received 3D axial $\mathrm{T} 1$ imaging $(\mathrm{TE} \sim 1.5 \mathrm{~ms}, \mathrm{TR} \sim 3.0 \mathrm{~ms}$, flip angle $\sim 10$ degrees) on the noncontrast, arterial, portovenous, 2-min, 5-min, 10-min, and 20-min delayed phases (Table 1). All patients received a 10-ml intravenous dose of gadoxetate at $1.0 \mathrm{mmol} / \mathrm{ml}$. Studies were performed on 1.5- (GE Twinspeed ${ }^{\mathrm{TM}}$ ) or 3.0-T (Philips Achieva $^{\mathrm{TM}}$ ) magnets with an eight-channel body phased array coil covering the entire liver. If a patient received multiple gadoxetate-enhanced MRIs prior to surgery, then the MRI performed closest to the date of surgery was used. We excluded patients who had their most recent MRI more than 9 months prior to surgery.

Demographic and clinical information was obtained on age, sex, number of liver metastases, size of the liver metastases, time from diagnosis of primary to diagnosis of liver metastases, node positivity of the primary colorectal cancer, and preoperative carcinoembryonic antigen (CEA) level within 6 months prior to surgery. The primary outcome of the study was overall survival post-MRI. We excluded patients who did not receive preoperative chemotherapy. We also excluded patients who died within 90 days of surgery to exclude deaths related to surgery.

The commonly used, validated, five-point, preoperative clinical risk score developed by Fong et al was calculated [11]. This score allots one point for each of five prognostic variables (number of liver metastases, size of the liver metastases, time from diagnosis of primary to diagnosis of liver metastases, node positivity of the primary colorectal cancer, and preoperative CEA level within 6 months prior to surgery) [11].

All clinical information was obtained using a prospectively maintained surgical database. Updated mortality data were obtained using electronic patient charts and publicly available obituary databases.

Table 1 MRI parameters for gadoxetate-enhanced MRI used in the study

MRI parameters

$\begin{array}{ll}\text { Plane } & \text { Axial } \\ \text { TE } & 1.5 \mathrm{~ms} \\ \text { TR } & 3.0 \mathrm{~ms} \\ \text { Flip angle } & 10 \text { degrees } \\ \text { Slice thickness } & 5 \mathrm{~mm} \\ \text { Spacing } & 2.5 \mathrm{~mm} \\ \text { Matrix } & 320 \times 192 \\ \text { Field of view } & 360 \mathrm{~mm} \\ \text { Contrast } & \text { Gadoxetate }(10 \mathrm{ml} \text { of } 1.0 \mathrm{mmol} / \mathrm{ml} \text { iv })\end{array}$




\section{Imaging analysis}

All imaging analysis was performed on the standard clinical picture archiving and communication system (PACS) software used at our institution (Agfa Impax 6.3.1, AGFA HealthCare N. V., Belgium $1^{\mathrm{TM}}$ ). CRCLMs were identified, and the signal-to-noise ratio (SNR) of each metastasis on noncontrast and 10- and 20-min delayed phases was calculated using previously described methods according to the following formula:

$S N R=\frac{\text { Signal intensity }(\text { tumour })}{\text { Standard deviation }(\text { noise })}$

Measurements of tumour signal intensity were performed at the axial level where the tumour was largest and by placing a round region of interest (ROI) most closely approximating the entire tumour. The background noise was calculated by taking eight 1-2-cm ROI samples surrounding the outside of the patient, but taking care to exclude banding due to motion artefact [4]:

The tumour enhancement at 10-min delayed phase (TuEn10) and 20-min delayed phase (TuEn20) for each CRCLM was calculated as the percentage increase in the SNR between the noncontrast and the 10-min and 20-min delayed phases, according to the following formula:

$T u E n X=\frac{S N R X-S N R 0}{S N R 0} \times 100 \%$

where SNRX is the signal-to-noise ratio of the liver metastasis at either 10- or 20-min delayed phase and SNR0 is the signal-to-noise ratio of the liver metastasis on noncontrast imaging.

If there were multiple lesions, then the two largest lesions were taken as target lesions and the mean TuEnX of the target lesion was taken as the target TuEnX, as described previously in the literature [4]. The mean TuEnX was used for survival analysis.

CRCLMs $<5 \mathrm{~mm}$ were excluded from analysis as they could not be accurately measured. Lesions were confirmed to be CRCLMs based on postoperative pathology reports.

\section{Survival analysis for target TuEnX}

The Youden Index was used to determine the value of Target TuEn10, which has the best sensitivity and specificity for predicting 3-year overall survival [12]. Target TuEn10 was dichotomised into strong and weak enhancement using the cut-off obtained from the Youden Index, which was used for survival analysis.

A chi-square test was used to determine if there were differences in demographic data between the strong and weak Target TuEn10 groups (Table 2).

Kaplan-Meier statistics were used to determine the univariate association between strong vs. weak Target TuEn10. Cox regression statistics were used to determine the multivariable association between strong vs. weak Target TuEn10 and overall survival after adjusting for clinical risk score.

Post-hoc sensitivity analyses were performed using Cox regression for any demographic variables that demonstrated significant differences between strong vs. weak Target TuEn10 (Table 2).

The analysis was repeated for Target TuEn20.

All analyses were performed on SPSS (IBM SPSS Statistics for Macintosh, version 22.0, 2013. Armonk, NY: IBM Corp.).

\section{Results}

\section{Patient population}

Sixty-five patients met inclusion/exclusion criteria for this study. Of 100 patients who had a preoperative gadoxetate-enhanced MRI prior to surgery for CRCLM, we excluded 8 where imaging was unavailable for analysis, 3 because of poor quality imaging, 4 who had portal vein embolisation prior to MRI, 2 who had undergone MRI more than 9 months prior to surgery, 11 who did not receive preoperative chemotherapy, and 7 patients who died within 90 days of surgery.

The average age of study patients was 61.5 years (SD: 12.4 years). There were 33 males (50.8\%) and 32 females (49.2\%). Baseline demographics are shown in Table 2. The time from MRI to surgery ranged from 0-9 months with a median time of 2.0 months.

\section{Survival analysis for target TuEn10}

Based on the Youden Index, the best threshold for predicting 3 -year mortality using Target TuEn10 was $25 \%$. Therefore, we dichotomised tumour enhancement into strong Target TuEn10 $(\geq$ $25 \%)$ and weak Target TuEn10 (<25\%). Thirty-six patients (with 5 events) had strong Target TuEn10 and 29 (with 13 events) had weak Target TuEn10 (Figs. 1 and 2).

Patient with strong Target TuEn10 were more likely to have smaller tumours $(p<0.03)$ and metachronous metastases $(p=$ $0.01)$. No other demographic data were significantly different between strong and weak Target TuEn10 groups (Table 2).

Sixty-five patients had data available for Kaplan-Meier analysis. On Kaplan-Meier analysis, strong Target TuEn10 was associated with overall survival (log-rank $p=0.001$ ) (Fig. 3). At 3 years (36 months), the proportion surviving was $85.1 \%$ (SE: $6.2 \%$ ) (number of events $=5$; number at risk $=27$ ) for those with strong Target TuEn10 compared with $56.5 \%$ (SE: $10.0 \%$ ) (number of events $=11$; number at risk $=13$ ) for those with weak Target TuEn10.

Sixty-three had data available for Cox regression analysis, in which strong Target TuEn10 was associated with overall 
Table 2 Baseline demographics for patients with strong vs. weak tumour enhancement of colorectal liver metastases on delayed phase gadoxetateenhanced MRI

\begin{tabular}{|c|c|c|c|c|c|c|}
\hline Description & $\begin{array}{l}\text { Weak target TuEn } 10 \\
(n=29)\end{array}$ & $\begin{array}{l}\text { Strong target TuEn } 10 \\
(n=36)\end{array}$ & $p$ value & $\begin{array}{l}\text { Weak target TuEn } 20 \\
(n=22)\end{array}$ & $\begin{array}{l}\text { Strong target TuEn20 } \\
(n=53)\end{array}$ & $p$ value \\
\hline \multicolumn{7}{|l|}{ Age, years } \\
\hline$<65$ & $15(51.7 \%)$ & $21(58.3 \%)$ & \multirow[t]{2}{*}{$p=0.59$} & $11(55.0 \%)$ & $25(56.8 \%)$ & \multirow[t]{2}{*}{$p=0.89$} \\
\hline$\geq 65$ & $14(48.3 \%)$ & $15(41.7 \%)$ & & $9(45.0 \%)$ & $19(43.2 \%)$ & \\
\hline \multicolumn{7}{|l|}{ Sex } \\
\hline Male & $12(41.4 \%)$ & $21(58.3 \%)$ & \multirow[t]{2}{*}{$p=0.17$} & $9(45.0 \%)$ & $23(52.3 \%)$ & \multirow[t]{2}{*}{$p=0.59$} \\
\hline Female & $17(58.6 \%)$ & $15(41.7 \%)$ & & $11(55.0 \%)$ & $21(47.7 \%)$ & \\
\hline \multicolumn{7}{|l|}{ Number of tumours } \\
\hline$\#$ of tumours $=1$ & $8(27.6 \%)$ & $13(36.1 \%)$ & \multirow[t]{2}{*}{$p=0.47$} & $6(30.0 \%)$ & $15(34.1 \%)$ & \multirow[t]{2}{*}{$p=0.75$} \\
\hline$\#$ of tumours $>1$ & $2172.4 \%)$ & $23(63.9 \%)$ & & $14(70.0 \%)$ & $29(65.9 \%)$ & \\
\hline \multicolumn{7}{|l|}{ Size of tumour } \\
\hline Size $\leq 5 \mathrm{~cm}$ & $22(75.9 \%)$ & $34(94.4 \%)$ & \multirow[t]{2}{*}{$p=0.03^{*}$} & $17(85.0 \%)$ & $38(86.4 \%)$ & \multirow[t]{2}{*}{$p=0.88$} \\
\hline Size $>5 \mathrm{~cm}$ & $7(24.1 \%)$ & $2(5.6 \%)$ & & $3(15.0 \%)$ & $6(13.6 \%)$ & \\
\hline \multicolumn{7}{|c|}{ Time from diagnosis of primary to diagnosis of liver metastases } \\
\hline Time $<12$ months & $11(37.9 \%)$ & $4(11.1 \%)$ & \multirow[t]{2}{*}{$p=0.01^{*}$} & $5(25.0 \%)$ & $10(22.7 \%)$ & \multirow[t]{2}{*}{$p=0.84$} \\
\hline Time $\geq 12$ months & $18(62.1 \%)$ & $32(88.9 \%)$ & & $15(75.0 \%)$ & $34(77.3 \%)$ & \\
\hline \multicolumn{7}{|l|}{ Node positive primary } \\
\hline \# of nodes positive $<5$ & $25(86.2 \%)$ & $28(77.8 \%)$ & \multirow[t]{2}{*}{$p=0.38$} & $17(85.0 \%)$ & $35(79.5 \%)$ & \multirow[t]{2}{*}{$p=0.60$} \\
\hline$\#$ of nodes positive $\geq 5$ & $4(13.8 \%)$ & $8(22.2 \%)$ & & $3(15.0 \%)$ & $9 \mathrm{c}(20.5 \%)$ & \\
\hline \multicolumn{7}{|c|}{ Preoperative carcinoembryonic antigen (CEA) } \\
\hline $\mathrm{CEA} \leq 200 \mathrm{ng} / \mathrm{ml}$ & $25(89.3 \%)$ & $34(94.4 \%)$ & \multirow[t]{2}{*}{$p=0.45$} & $17(85.0 \%)$ & $41(95.3 \%)$ & \multirow[t]{2}{*}{$p=0.16$} \\
\hline $\mathrm{CEA}>200 \mathrm{ng} / \mathrm{ml}$ & $3(10.7 \%)$ & $2(5.6 \%)$ & & $3(15.0 \%)$ & $2(4.7 \%)$ & \\
\hline Data not available & 1 & & & & & \\
\hline \multicolumn{7}{|l|}{ Clinical risk score } \\
\hline$<3$ & $19(70.4 \%)$ & $30(83.3 \%)$ & \multirow[t]{2}{*}{$p=0.22$} & $14(77.8 \%)$ & $34(77.3 \%)$ & \multirow[t]{2}{*}{$p=0.97$} \\
\hline$\geq 3$ & $8(29.6 \%)$ & $6(16.7 \%)$ & & $4(22.2 \%)$ & $10(22.7 \%)$ & \\
\hline Data not available & 2 & & & & & \\
\hline \multicolumn{7}{|l|}{ Magnet } \\
\hline $1.5 \mathrm{~T}$ & $2(6.9 \%)$ & $8(22.9 \%)$ & \multirow[t]{2}{*}{$p=0.08$} & $3(15.0 \%)$ & $7(15.9 \%)$ & \multirow[t]{2}{*}{$p=0.93$} \\
\hline $3.0 \mathrm{~T}$ & $27(93.1 \%)$ & $27(77.1 \%)$ & & $17(85.0 \%)$ & $37(84.1 \%)$ & \\
\hline
\end{tabular}

$* p<0.05$

survival ( $p=0.009$ ), independent of clinical risk score. The adjusted hazard ratio of Target TuEn10 and clinical risk score for overall survival were 0.24 (95\% CI: $0.08-0.70)$ and 2.61 (95\% CI: 0.98-6.98) respectively (Table 3 ).

Post-hoc sensitivity analyses were performed for synchronous vs. metachronous metastases. When tumour size and synchronous metastases were included in the Cox regression model, Target TuEn10 had an adjusted hazard ratio of 0.23 (95\% CI: 0.07-0.72).

\section{Survival analysis for target TuEn20}

Based on the Youden Index, the best threshold for predicting 3-year mortality using Target TuEn20 was 77\%. Therefore, we
Fig. 1 Pathology-confirmed colorectal liver metastases on preoperative 3D Axial T1 MRI at (a) precontrast, (b) 10-min postgadoxetate injection, and (c) 20min post-gadoxetate injection in a 60-year-old male demonstrating strong tumour enhancement

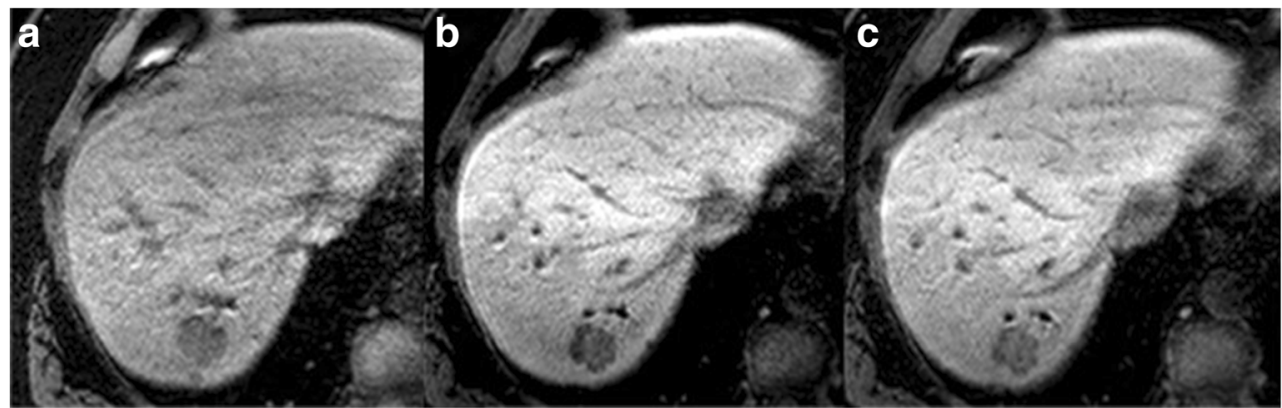


Fig. 2 Pathology-confirmed colorectal liver metastases on preoperative 3D axial T1 MRI at (a) precontrast, (b) 10-min postgadoxetate injection, and (c) 20min post-gadoxetate injection in a 69-year-old male demonstrating weak tumour enhancement

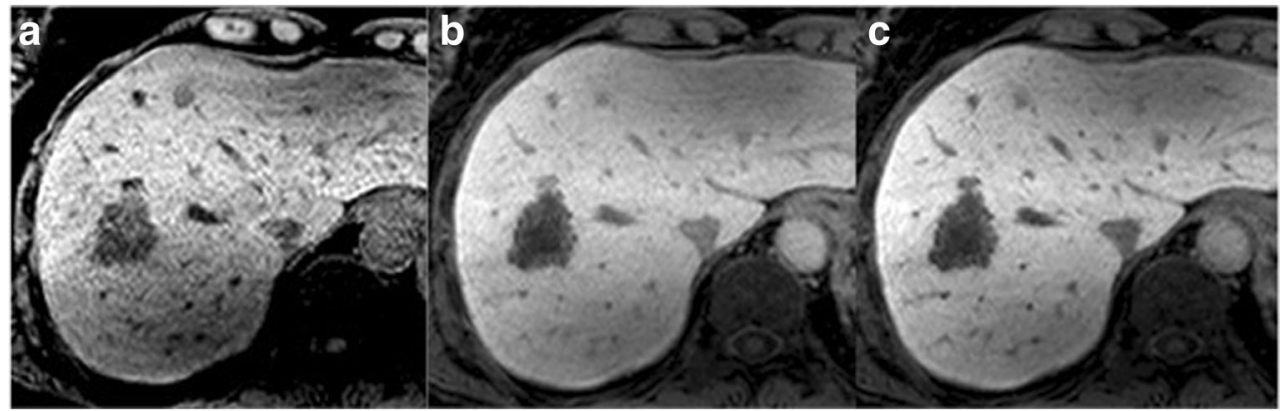

dichotomised tumour enhancement into strong Target TuEn20 $(\geq 77 \%)$ and weak Target TuEn20 (<77\%). Forty-four patients (with 8 events) had strong Target TuEn20 and 20 patients (with 10 events) had weak Target TuEn20 (Figs. 1 and 2).

No demographic data were significantly different between strong and weak Target TuEn20 groups (Table 2).

Sixty-four patients had data available for Kaplan-Meier analysis, in which strong Target TuEn20 was associated with overall survival (log-rank $p=0.011$ ) (Fig. 4). At 3 years $(36$ months), the proportion surviving was $79.4 \%$ (SE: 6.5\%) (number of events $=8$; number at risk $=29$ ) for those with strong Target TuEn20 compared with $58.7 \%$ (SE: 11.2\%) (number of events $=8$; number at risk $=10$ ) for those with weak Target TuEn20.

Sixty-two 62 patients had data available for Cox regression analysis, in which strong Target TuEn20 was associated with overall survival ( $p=0.018$ ), independent of clinical risk score.

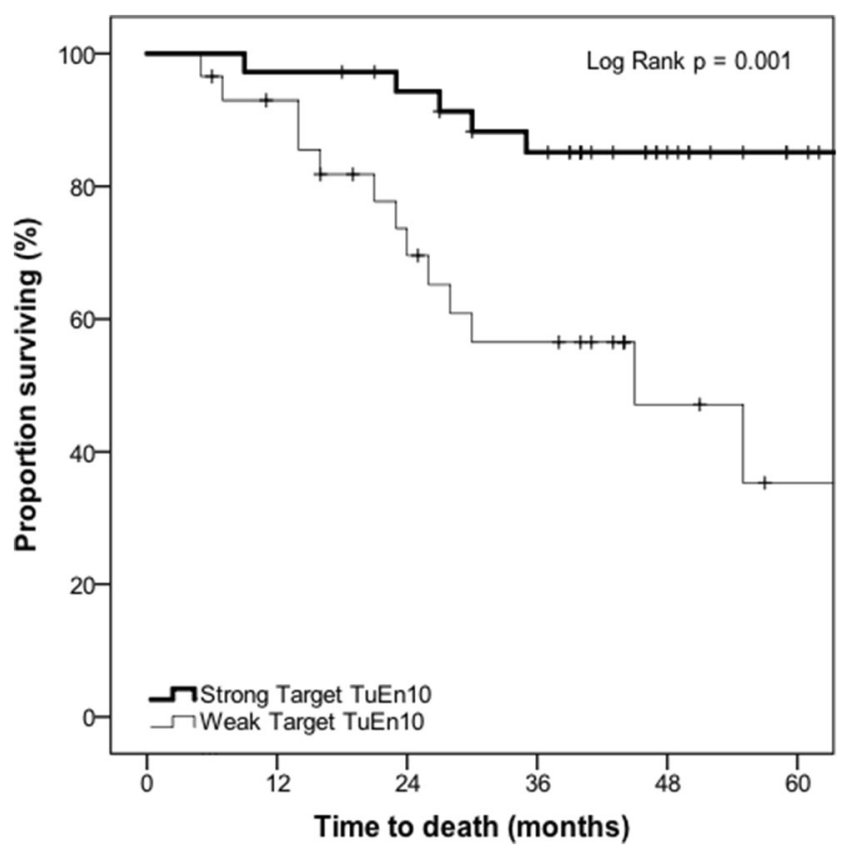

Fig. 3 Kaplan-Meier survival curve demonstrating the association between target tumour enhancement at 10-min delayed phase (Target TuEn10) and overall survival in patients with colorectal liver metastases who had received preoperative gadoxetate-enhanced MRI prior to hepatectomy $(n=65)(p=0.001)$
The adjusted hazard ratio of Target TuEn20 and clinical risk score for overall survival were 0.32 (95\% CI: 0.12-0.82) and 3.20 (95\% CI: 1.21-8.45) respectively (Table 4).

\section{Discussion}

This study provides preliminary evidence that tumour enhancement of CRCLM on preoperative gadoxetate-enhanced MRI is associated with survival post-hepatectomy, after adjusting for known confounders. In patients who had strong target tumour enhancement on 10-min delayed phase, the proportion surviving at 36 months was $85.1 \%$ compared with $56.5 \%$ in patients who had weak target tumour enhancement, an absolute difference in overall survival of $28.6 \%$. The adjusted hazard ratio, after considering known confounders, was 0.24. In patients who had strong target tumour enhancement

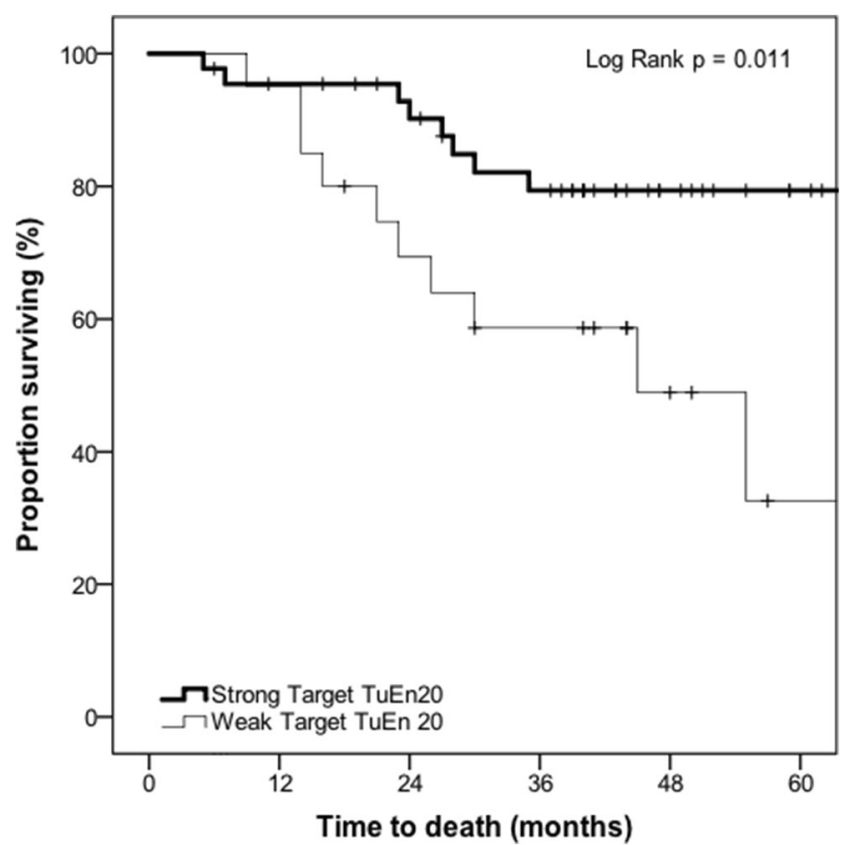

Fig. 4 Kaplan-Meier survival curve demonstrating the association between target tumour enhancement at 20-min delayed phase (Target TuEn20) and overall survival in patients with colorectal liver metastases who had received preoperative gadoxetate-enhanced MRI prior to hepatectomy $(n=64)(p=0.011)$ 
Table 3 Cox regression model for adjusted hazard ratios for the association of target tumour enhancement at 10-min delayed phase imaging (Target TuEn10) on gadoxetate-enhanced MRI and overall survival $(n=63)$

\begin{tabular}{lll}
\hline & $\begin{array}{l}\text { Adjusted hazard ratio } \\
\text { (95\% confidence interval) }\end{array}$ & $p$ value \\
\hline Target TuEn10 & & \\
$\quad$ Weak & Reference & $p=0.009^{* *}$ \\
$\quad$ Strong & $0.24(0.08-0.70)$ & \\
Clinical risk score & & $p=0.055$ \\
$<3$ & Reference & \\
$\geq 3$ & $2.61(0.98-6.98)$ & \\
\hline
\end{tabular}

$* * p<0.01$

on 20-min delayed phase, the proportion surviving at 36 months was $79.4 \%$ compared with $58.7 \%$ in patients who had weak target tumour enhancement, an absolute difference in overall survival of $20.7 \%$. The adjusted hazard ratio, after taking into account known confounders, was 0.32 . Therefore, tumour enhancement on delayed phase gadoxetate-enhanced MRI may represent a prognostic imaging biomarker of survival in this patient population.

Prior studies with extracellular contrast agents have demonstrated that late gadolinium enhancement is associated with tumour fibrosis, which is a known predictor of good outcomes post-hepatectomy in patients with CRCLM [4] . Future prospective, high-resolution, matched radiological-pathological correlation studies are required to determine whether this is also the case with gadoxetate. Prior studies with hepatobiliary-specific contrast agents have suggested that late gadolinium enhancement may be related to OATP1B3 expression and treatment response in a chemotherapy-naïve population [13, 14].

All patients were surgical candidates who received preoperative chemotherapy as per institutional clinical guidelines. However, in this retrospective cohort, the exact amount and type of chemotherapy varied between patients. This could

Table 4 Cox regression model for adjusted hazard ratios (HRs) for the association of target tumour enhancement at 20-min delayed phase imaging (Target TuEn20) on gadoxetate-enhanced MRI and overall survival $(n=62)$

\begin{tabular}{lll}
\hline & $\begin{array}{l}\text { Adjusted hazard ratio } \\
\text { (95\% confidence interval) }\end{array}$ & $p$ value \\
\hline Low TuEn 20 & & \\
$\quad$ Weak & Reference & $p=0.018^{*}$ \\
$\quad$ Strong & $0.32(0.12-0.82)$ & \\
Clinical risk score & & $p=0.019^{*}$ \\
$<3$ & Reference & \\
$\geq 3$ & $3.20(1.21-8.45)$ & \\
\hline
\end{tabular}

$* p<0.05$ affect outcomes and the results of this study. Further prospective studies controlling for chemotherapy regimens are required.

Although there is currently no literature on the timing of delayed phase imaging and correlation with tumour fibrosis in CRCLM, prior studies in the cardiac literature demonstrated that late gadolinium enhancement is best correlated with tissue fibrosis between 10-30 min using extracellular contrast agents [10]. In our study, tumour enhancement was correlated with overall survival at both 10- and 20-min delayed phases. Due to the small sample size, we were unable to directly compare tumour enhancement at 10- versus 20-min delayed phases and it is unclear based on our study if one time is better than the other or if both time points are required for evaluation of tumour enhancement. In this study, we specifically correlated delayed phase tumour enhancement with outcomes based on the prior literature [4]; however, it is possible that tumour enhancement at other phases (e.g. arterial, portovenous, or 2-min delayed phases) may provide additional prognostic information. This could be explored in future studies.

The ability to assess multiple tumours may be one of the significant advantages of imaging biomarkers over pathology and molecular biomarkers. There is growing evidence that tissue sampling is prone to error due to tumour heterogeneity among different tumours and even within the same tumour [15-17]. In heterogeneous tumour clones, sampling of tumours may not accurately measure the most aggressive tumour, which may be most likely to drive patient survival [18]. Additionally, pathology and molecular prognostic biomarkers cannot be easily assessed preoperatively. Evaluation of pathology requires tissue specimens, which may be difficult to obtain. Liver biopsy is often not feasible because of technical difficulty as well as the risk of needle tract seeding [19] . The proposed MRI biomarker addresses some of these limitations, since it is a cross-sectional technique that can identify and evaluate all tumours and, unlike current pathology or molecular biology techniques, it does not require tumour sampling.

Prognostic biomarkers may be particularly important in CRCLM because of the heterogeneity in outcomes in this patient population. It is increasingly understood that patients who have "good" biology may respond well to surgery even with features that were traditionally considered contraindications such as R1 resection or extrahepatic disease [19]. However, it is less clear how to determine preoperatively which patients have "good" biology and will benefit from treatment. Risk-stratification using prognostic biomarkers may be the key to addressing this challenge.

In our study, tumour enhancement at 10-min delayed phase was also associated with smaller tumour size and metachronous metastases. This may be because tumour enhancement is a good prognostic variable and was therefore associated with other known good prognostic variables [11]. 
In our post-hoc sensitivity analysis, tumour enhancement at 10-min delayed phase was associated with good outcomes independent of tumour size and the presence of metachronous metastases.

Gadoxetate-enhanced MRI is already routinely used in the preoperative diagnosis and staging of patients undergoing surgery for CRCLM, and 10- and 20-min delayed phase imaging is routinely performed on clinical gadoxetate-enhanced MRI [7]. This technique is widely available and measuring tumour enhancement is straightforward, does not require significant training, and can be done on standard clinical PACS software available at almost all cancer institutions that offer MRI. Therefore, if this imaging biomarker can be externally validated in future studies, it may be easily adapted for clinical practice.

There are a number of limitations to this study. This study was a small, preliminary, retrospective study. Larger multicentre studies are required to replicate and validate the results. In addition, prospective studies are required to determine how this finding may be used clinically for patient management. Further studies are also required to optimise techniques for measuring MRI enhancement.

In conclusion, tumour enhancement on gadoxetate-enhanced MRI is associated with long-term survival in patients with colorectal liver metastases and may represent a biomarker of prognosis.

Funding The authors state that this work has not received any funding.

\section{Compliance with ethical standards}

Guarantor The scientific guarantor of this publication is Dr. Laurent Milot.

Conflict of interest The authors of this manuscript declare no relationships with any companies, whose products or services may be related to the subject matter of the article.

Statistics and biometry No complex statistical methods were necessary for this paper.

Informed consent Written informed consent was waived by the Institutional Review Board.

Ethical approval Institutional Review Board approval was obtained.

\section{Methodology \\ - retrospective \\ - cross-sectional study \\ - performed at one institution}

Open Access This article is distributed under the terms of the Creative Commons Attribution 4.0 International License (http://creativecommons. org/licenses/by/4.0/), which permits unrestricted use, distribution, and reproduction in any medium, provided you give appropriate credit to the original author(s) and the source, provide a link to the Creative Commons license, and indicate if changes were made.

\section{References}

1. World Health Organization (2018) WHO Cancer Fact Sheet. World Health Organization, Geneva. Available via http://www.who.int/en/ news-room/fact-sheets/detail/cancer. Accessed 29 April 2018

2. Kanas GP, Taylor A, Primrose JN et al (2012) Survival after liver resection in metastatic colorectal cancer: review and meta-analysis of prognostic factors. Clin Epidemiol 4:283-301

3. Collins D, Chua H (2017) Contemporary surgical management of synchronous colorectal liver metastases. F1000Res 6:598

4. Cheung HMCKP, Hsieh E, Coburn N et al (2018) Late gadolinium enhancement of colorectal liver metastases post-chemotherapy is associated with tumour fibrosis and overall survival post-hepatectomy. Eur Radiol. https://doi.org/10.1007/s00330-018-5331-4

5. Poultsides GA, Bao F, Servais EL et al (2012) Pathologic response to preoperative chemotherapy in colorectal liver metastases: fibrosis, not necrosis, predicts outcome. Ann Surg Oncol 19(9):2797-2804

6. Rubbia-Brandt L, Giostra E, Brezault C et al (2007) Importance of histological tumor response assessment in predicting the outcome in patients with colorectal liver metastases treated with neoadjuvant chemotherapy followed by liver surgery. Ann Oncol 18 (2):299-304

7. Seale MK, Catalano OA, Saini S, Hahn PF, Sahani DV (2009) Hepatobiliary-specific MR contrast agents: role in imaging the liver and biliary tree. Radiographics 29(6):1725-1748

8. Granata V, Catalano O, Fusco R et al (2015) The target sign in colorectal liver metastases: an atypical Gd-EOB-DTPA "uptake" on the hepatobiliary phase of MR imaging. Abdom Imaging 40 (7):2364-2371

9. Kim A, Lee $\mathrm{CH}, \mathrm{Kim} \mathrm{BH}$ et al (2012) Gadoxetic acid-enhanced 3.0T MRI for the evaluation of hepatic metastasis from colorectal cancer: metastasis is not always seen as a "defect" on the hepatobiliary phase. Eur J Radiol 81(12):3998-4004

10. Oshinski JN, Yang Z, Jones JR, Mata JF, French BA (2001) Imaging time after Gd-DTPA injection is critical in using delayed enhancement to determine infarct size accurately with magnetic resonance imaging. Circulation 104(23):2838-2842

11. Fong Y, Fortner J, Sun RL, Brennan MF, Blumgart LH (1999) Clinical score for predicting recurrence after hepatic resection for metastatic colorectal cancer: analysis of 1001 consecutive cases. Ann Surg 230(3):309-318 discussion 18-21

12. Youden WJ (1950) Index for rating diagnostic tests. Cancer 3(1):32-35

13. Murata S, Matsushima S, Sato Y et al (2018) Predicting chemotherapeutic response for colorectal liver metastases using relative tumor enhancement of gadoxetic acid disodium-enhanced magnetic resonance imaging. Abdom Radiol (NY)

14. Park SH, Kim H, Kim EK et al (2017) Aberrant expression of OATP1B3 in colorectal cancer liver metastases and its clinical implication on gadoxetic acid-enhanced MRI. Oncotarget 8(41): 71012-71023

15. Gerlinger M, Rowan AJ, Horswell S et al (2012) Intratumor heterogeneity and branched evolution revealed by multiregion sequencing. N Engl J Med 366(10):883-892

16. Swanton C (2012) Intratumor heterogeneity: evolution through space and time. Cancer Res 72(19):4875-4882

17. Punt CJ, Koopman M, Vermeulen L (2017) From tumour heterogeneity to advances in precision treatment of colorectal cancer. Nat Rev Clin Oncol 14(4):235-246

18. Jamal-Hanjani M, Quezada SA, Larkin J, Swanton C (2015) Translational implications of tumor heterogeneity. Clin Cancer Res 21(6): 1258-1266

19. Jones OM, Rees M, John TG, Bygrave S, Plant G (2005) Biopsy of resectable colorectal liver metastases causes tumour dissemination and adversely affects survival after liver resection. Br J Surg 92(9): $1165-1168$ 